\title{
CODEPOSICIÓN ELECTROLÍTICA DE ZINC Y NÍQUEL, CON FINES DE MEJORAR EL GALVANIZADO INDUSTRIAL ESTÁNDAR
}

\section{ELECTROLYTIC CODEPOSITION OF ZINC AND NICKEL, IN ORDER TO IMPROVE STANDARD INDUSTRIAL GALVANIZING

\author{
Ángel Villón-Ulloa ${ }^{1 *} \mathbb{C}$, Jesús Cruz-Contreras ${ }^{10}$
}

'Universidad Nacional de Ingeniería, Facultad de Ingeniería Química y Textil, Lima, Perú

Recibido (Received): 09/10/2020 Aceptado (Accepted): 30/06/2021

\begin{abstract}
RESUMEN
Se logró un galvanizado mejorado con la codeposición de Zn y Ni, mejorando la dureza a valores de 140-148 HV y una velocidad de corrosión de 4.8-6.4 mpy con un depósito de 9.8\% de Ni y 90.2\% de zinc, con un baño de sales de sulfato de $\mathrm{Ni} \mathrm{y} \mathrm{Zn} \mathrm{a} \mathrm{pH} \mathrm{1-3,}$ usando como aditivos tiourea, gluconato de sodio y ácido bórico entre otros, con densidades de corriente diferenciados para cada ánodo, de níquel y el de zinc, corrientes totales de 5-6 A/dm2, los tiempos utilizados fueron los suficientes para provocar un espesor adecuado para ensayos de 10-15 minutos, con una celda electrolítica con agitación, con eficiencia catódicas de 48 a $65 \%$.
\end{abstract}

Palabras Clave: Electrocodeposición zinc/níquel, galvanizado mejorado, velocidad de corrosión

\section{ABSTRACT}

An improved galvanizing with the $\mathrm{Zn}$ and $\mathrm{Ni}$ co-deposition, achieved an improvement in the hardness at $140-148 \mathrm{HV}$ and a corrosion rate of 4.8-6.4 mpy with a deposit of $9.8 \% \mathrm{Ni}$ and $90.2 \%$ of $\mathrm{Zn}$, with a salt bath of $\mathrm{Ni}$ and $\mathrm{Zn}$ sulphate at $\mathrm{pH} 1-3$, using as additives thiourea, sodium gluconate and boric acid among others, with differentiated current densities for each anode, nickel with $6 \mathrm{~A} / \mathrm{dm}^{2}$ and for zinc $5 \mathrm{~A} / \mathrm{dm}^{2}$, the times used were enough to cause an adequate thickness for tests of 10-15 minutes, with an electrolytic cell with agitation, with cathodic efficiency of 48 to $65 \%$.

Keywords: Electrocodeposition zinc/ nickel, improved galvanization, corrosion rate

\section{INTRODUCCIÓN}

La codeposición es una técnica electroquímica utilizada para que se produzca en forma simultánea la reducción de dos o más metales sobre una superficie base, en términos sencillos crear una aleación. En el caso del galvanizado mejorado, deberá contar junto con el zinc otro metal que pueda mejorar las propiedades del recubrimiento del zinc, como la dureza y la protección contra la corrosión, las velocidades de corrosión normales para el zinc como galvanizado están en el orden de $50 \mathrm{mpy}$, por lo tanto, mejorar (reducir) este valor ya sería un beneficio de la codeposición protectora. Se han probado con diferentes metales cobalto, níquel, cadmio, boro $[1,2]$.

\footnotetext{
1* Corresponding author:
}

E-mail: avillon@uni.edu.pe
Debido a temas de toxicidad, de disponibilidad o costos, el níquel es el metal que se utilizó como elemento para mejorar el galvanizado estándar, en esta investigación. El níquel es un metal que se considera noble y por lo tanto muy estable en procesos de corrosión, además es un metal que se pasiva (forma una capa protectora, adherente, no soluble y no conductora) por lo tanto candidato a proteger contra la corrosión, además ofrece una mejora en la dureza (níquel dureza Vickers $356 \mathrm{Hv}$ y zinc $45 \mathrm{Hv}$ [3]) de la superficie y esto mejora el galvanizado estándar.

La electrodeposición de un solo metal depende de las siguientes variables: composición del baño (tipo y cantidad de las sales involucradas y del $\mathrm{pH}$ ), aditivos involucrados (retardadores y abrillantadores) y de las condiciones de operación, como la densidad de corriente utilizada, temperatura y agitación del baño. A esta problemática para un solo elemento electrodepositado, en una codeposición se suma otros factores más, por ejemplo, la composición adecuada de los metales aleantes en el recubrimiento, además que la deposición suceda simultáneamente $y$ en las 
proporciones requeridas, creando una metalografía (estructura cristalina) deseada, debido a que las características protectoras del recubrimiento, dependerá de todos estos factores.

Los tipos de baño para las codeposiciones de zinc/níquel son variadas, pudiendo ser estás en base a sales de sulfato, sales de cloruros (generalmente ligeramente ácidas) y las llamadas alcalinas (debido a su $\mathrm{pH}$ y el uso de óxidos en su composición), además se incluye el uso de aditivos y estos pueden ser del tipo retardadores o abrillantadores independientemente del tipo de baño utilizado. Los retardadores son especies químicas que tienen como función retardar la velocidad de reducción de la forma iónica del metal y de esta manera contribuir a una creación de una estructura cristalina ordenada (para que no tenga la apariencia oscura de "quemado") y los abrillantadores van por lo mismo, son especies químicas que acompañan al ion hasta la superficie del cátodo, colocándolo en la posición adecuada y de esta manera se obtiene una estructura cristalina sin sitios de vacancia, contribuyendo a una superficie más lisa, más compacta y de esta manera contribuyendo al brillo de la superficie y a su dureza.

\subsection{CONSIDERACIONES}

PREVIAS EXPERIMENTALES

Las condiciones de operación como la densidad de corriente, es de suma importancia, porque define muchas características: (1) el nivel de polarización y con esto la eficiencia catódica (parámetro que mide la fracción de la corriente en la reducción utilizada por las especies deseadas), (2) la velocidad de codeposición (orden del acomodo de los átomos reducidos), (3) también define el potencial eléctrico del cátodo (define la simultaneidad de la codeposición), (4) define la proporción de los metales a depositarse, (5) como también la forma como lo hacen, creando sistemas cristalinos diferentes (fases), los cuales definirán las propiedades químicas (corrosión) y físicas del recubrimiento (dureza), por tales razones la densidad de corriente juega un papel muy importante en la codeposición, de zinc y níquel.

El potencial eléctrico que se alcanza en los electrodos tanto ánodo y cátodo dependerá de la densidad de corriente circulada por el sistema y de la polarización, este potencial funciona como un umbral energético para que ciertas especies iónicas se oxiden o reduzcan, de tal manera que el potencial eléctrico define si el zinc y el níquel se codepositarán, además definirá la forma como se alcanzará el arreglo cristalino, la fase que deberá presentarse es la fase ? (gamma) según Abou-Krisha, Moonjae Kwon, Soroor Ghaziof y Bahadormanesh $[3,4,5,6,7]$, para esto se busca una proporción que está en el orden de 10-12\% de níquel
(Ni5Zn21) $[1,3,5,7,8,9,10,11]$ la cual proporciona la mejor protección contra la corrosión, la proporción del níquel no debe superar ese rango porque se ha comprobado que si eso sucede el recubrimiento se comportará como cátodo y la base de acero comercial se comportaría como ánodo, invirtiéndose la función protectora del recubrimiento.

El potencial no sólo es una función de la densidad de corriente, sino además de un fenómeno que se crea en el sistema conocido como polarización (por concentración, por activación o por resistencia) que hace que el potencial varié a pesar de tener una misma densidad de corriente, este fenómeno origina una caída del potencial en el cátodo. La importancia del valor del potencial reside en si se encuentra un potencial asociado a un recubrimiento exitoso, este dato serviría para reproducir las mismas características del recubrimiento, utilizando un baño similar, usando el mismo potencial. Para que la polarización por concentración no afecte el potencial, se utiliza la agitación lo cual reduce el efecto de la polarización de este tipo y de esta manera la eficiencia catódica no se ve disminuida por la polarización.

Para evaluar la velocidad de corrosión se usaron los métodos electroquímicos, como el método conocido como intercepto (pendientes de Tafel) y el método de RPL (resistencia de polarización) ambos métodos se utilizó un potenciostato marca Gamry Interface1010 T.

Para generar la corriente continua se utilizó el equipo rectificador de corriente autoregulable con tres salidas independientes de corriente, marca Singlet, de 6.4 amperios/32 voltios.

Para determinar la estructura y fases del recubrimiento $\mathrm{Zn}-\mathrm{Ni}$ se usó la técnica Difracción de Rayos X, Difractómetro de Rayos X Philips X Pert Pro del Centro de Desarrollo de Materiales Avanzados y Nanotecnología, Facultad de Ciencias-UNI.

Para determinar la composición del recubrimiento (\% de $\mathrm{Ni}$ y \% de $\mathrm{Zn}$ ) se utilizó la técnica de absorción atómica, con el equipo Espectrofotómetro de Absorción Atómica Savanta AA - GBC. (Facultad de Ingeniería Geológica, Minera y Metalúrgica, Laboratorio de Espectrometría).

La microdureza del recubrimiento $\mathrm{Zn} / \mathrm{Ni}$ se midió usando un Microdurómetro marca MH HVS 1000 A CARL ZEISS JENA a partir de probetas de $2 \mathrm{~cm} \times 3 \mathrm{~cm}$ aplicando una carga de 100 g por $15 \mathrm{~s}$ por diferentes zonas se midió reportando microdureza Vickers, con la siguiente relación.

$H V=\frac{1.854 P}{d^{2}}$ 
Donde $\mathrm{d}$ es la diagonal promedio de la superficie de la impronta y $\mathrm{P}$ la carga aplicada en $\mathrm{kg}$.

La eficiencia catódica se calculó usando la ley de Faraday:

$$
E f i=\frac{\text { Peso experimental del depósito }}{\text { Peso teórico del depósito(Faraday })}
$$

Si no se conoce la composición de $\mathrm{Zn} / \mathrm{Ni}$ del depósito, se asume que hay un depósito de $90 \%$ de $Z n$ y $10 \%$ de Ni, para evaluar el peso teórico.

\section{ANTECEDENTES}

Hay en la literatura algunas propuestas de codeposición de diferentes metales lo que incluye cobalto, boro y níquel, con el zinc, todas buscan mejorar las propiedades anticorrosivas del zinc, y alguna otra propiedad del mismo. [24]

Los artículos se basan en ofrecer las condiciones de baño y condiciones de operación primero para una codeposición exitosa, la mayoría usa baños alcalinos cianurados, que el baño pueda lograr la composición del zinc/níquel adecuada para tener las propiedades esperadas. [25]

Como también que aditivos podrían usarse en la composición del baño básico, y ofrecer algunas características metalográficas que redundarían sobre las propiedades del recubrimiento. [26]

Sobre las pruebas electroquímicas para evaluar las propiedades anticorrosivas de la codeposión, hay material extenso y claro, además forma parte de los temas ensayados en los laboratorios de corrosión.

\section{METODOLOGÍA}

La configuración del ensayo experimental consistió en una celda de polietileno de 2.3 I de capacidad, (se usó agua osmótica $5 \mu \mathrm{S} / \mathrm{cm}$ ), para albergar dos ánodos de zinc calidad electrolítica $99.99 \%$ de pureza y dos ánodos de níquel calidad electrolítica $99.99 \%$ de pureza, en el medio de la celda la probeta o cupón de prueba $(5 \times 10$ $\mathrm{cm}, 1 \mathrm{dm}^{2}$ ) que inicialmente fue de bronce (por ser un material inerte) y luego se cambió a una probeta de acero comercial, debido a que este material es más utilizado en un galvanizado. Se utilizó agitación en las pruebas, usando un agitador magnético.

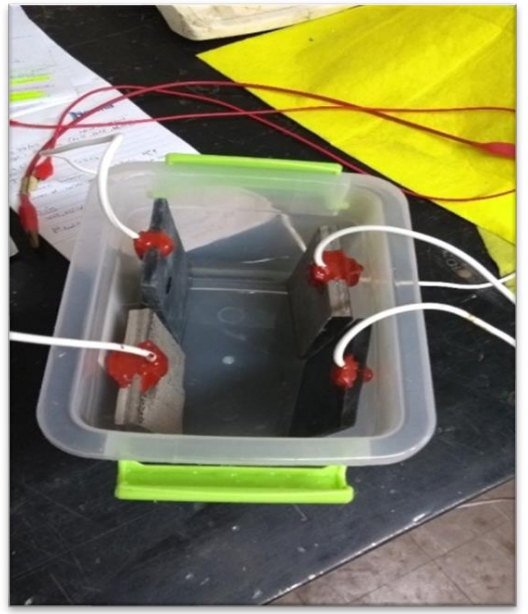

Fig. 1. Disposición de los ánodos de níquel y zinc

La parte experimental de la investigación tuvo tres objetivos (1) encontrar el tipo y composición del baño que permita la codeposición [4,15], (2) encontrar los aditivos adecuados (retardador y abrillantador) y las cantidades adecuadas, (3) la densidad de corriente diferenciada (para los ánodos de zinc y níquel) que permita el tipo de depósito y porcentaje buscado de Zn y $\mathrm{Ni}$.

Inicialmente para la búsqueda del tipo de baño, no se usaron aditivos, para que de esta manera verificar las ventajas o desventajas del tipo de baño sin la contribución de especies químicas ajenas al mismo baño. Se comenzó utilizando baños de cloruros con la siguiente composición $\mathrm{ZnCl} 238.16 \mathrm{~g} / \mathrm{l}$ y NiCl $2.6 \mathrm{H}_{2} \mathrm{O}$ $35.66 \mathrm{~g} / \mathrm{l} \mathrm{pH}$ 5, luego se utilizó el baño de sulfatos con la siguiente composición $\mathrm{ZnSO} 4.7 \mathrm{H}_{2} \mathrm{O} 143.78 \mathrm{~g} / \mathrm{l}$ y $\mathrm{NiSO} 4.6 \mathrm{H}_{2} \mathrm{O} 52.57 \mathrm{~g} / \mathrm{l} \mathrm{pH} 3.8$ y por último un baño alcalino con la siguiente composición NiSO4.6 $\mathrm{H}_{2} \mathrm{O} 24.16$ $\mathrm{g} / \mathrm{l}, \mathrm{ZnO} 10 \mathrm{~g} / \mathrm{l}$ y NaOH $100 \mathrm{~g} / \mathrm{l} \mathrm{pH}$ 13. Todos los productos químicos usados son de calidad QP (químicamente puro, con pureza por encima del 99\%)El baño de cloruros ocasiono formación de óxidos de níquel de color negro sobre los ánodos de níquel, en forma espontánea, sin el uso de corriente, lo cual originaba la incapacidad de este ánodo de suplir de iones de níquel al medio, por lo tanto, se descartó este tipo de baño.

El baño de sulfatos no ocasiono problemas operativos, ni depósitos extraños, no afecto a los electrodos tanto ánodos o cátodos y se obtuvo depósitos bastante aceptables. El baño alcalino 
ocasiono problemas de deposición muy rugosa, oscura, con material reducido no adherente (arborización). Por lo tanto, el baño más se adecuó a los objetivos fue el baño de sulfatos, el pH de este baño se regulaba usando ácido sulfúrico en la cantidad necesaria.

Para la búsqueda de los aditivos teníamos la bibliografía $[1,7,10,12,13]$ que nos sugería el uso de los siguientes agentes químicos, ácido bórico, tiourea, sacarina, propilenglicol, EDTA, gluconato de sodio. Los retardadores ocasionan una disminución de la velocidad de reducción de los iones, en algunos casos formando complejos o especies químicas reteniendo el ion usando para ello los grupos funcionales que dispone el retardador, permitiendo de esta manera una deposición más uniforme y los abrillantadores, producen una superficie lisa y más compacta.

Se utilizaron las cantidades (pesos) recomendados en la bibliografía $[12,13,19]$, como retardadores se usaron tiourea, sacarina, propilenglicol, EDTA, gluconato de sodio y como abrillantador fue el ácido bórico, posteriormente se modificaron las cantidades inicialmente recomendada en la bibliografía, esta variación dependía del éxito (apariencia, eficiencia del recubrimiento), algunas de esas cantidades usadas en los baños de sulfatos se observa en la tabla I, las variaciones de los pesos en relación a la tiourea (retardador para níquel) se justificaban de la siguiente manera, un aumento del peso reduciría el contenido de níquel en el recubrimiento, como se pudo comprobar en la figura 2, en relación al gluconato de sodio (retardador para zinc), el efecto se aprecia en la figura 3 , se puede observar que hay un efecto significativo a $6 \mathrm{~g} / \mathrm{l}$ sobre la presencia de níquel. Las técnicas utilizadas para generar las figuras 2 y 3 , como no existe modelos o teoría sobre el efecto de que tiene cada aditivo sobre el peso \% de $\mathrm{Ni}$ en el recubrimiento, fue prueba y error, evidenciando el efecto del incremento de peso y midiendo si efecto en el \% de $\mathrm{Ni}$ en el recubrimiento.

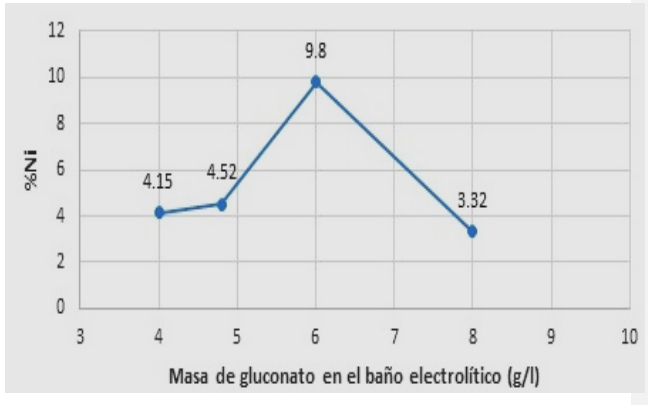

Fig. 3. Efecto del gluconato de sodio en el contenido de níquel en el recubrimiento

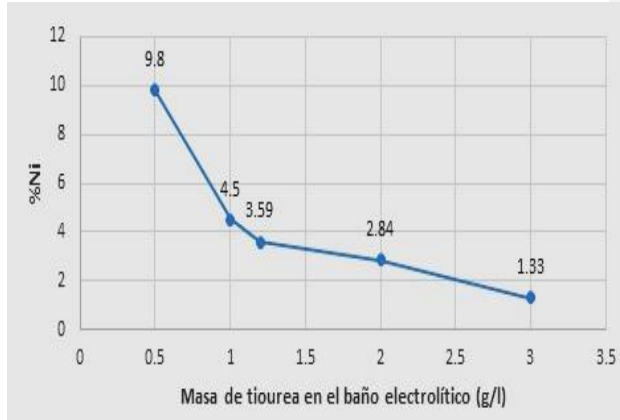

Fig. 2. Efecto de la tiourea en el contenido de níquel en el recubrimiento

Una vez ya definido el tipo de baño (sulfatos), lo siguiente fue definir las relaciones molares de $\mathrm{Zn} / \mathrm{Ni}$, en el baño, lo cual definiría el \% de $\mathrm{Ni}$, los resultados más exitosos se aprecian en la tabla II, estas pruebas consideraron dentro de la composición del baño la participación de aditivos (abrillantador/retardador), los cuales a su vez se hicieron variaciones en sus pesos en los baños de prueba, para observar el efecto sobre la composición de la electrodeposición. 
TABLA I

Composición de baños de sulfato y el uso de aditivos

\begin{tabular}{|c|c|c|c|c|}
\hline Prueba & Composición del baño (g/l) & $\mathrm{pH}$ & Relación molar de Zn/Ni en el baño & $\begin{array}{c}\text { Densidad de } \\
\text { corriente }\left(\mathrm{A} / \mathrm{dm}^{2}\right)\end{array}$ \\
\hline 164 & $\begin{array}{l}\mathrm{ZnSO}_{4.7} \mathrm{H}_{2} \mathrm{O}(287.55 \mathrm{~g} / \mathrm{l}) \\
\mathrm{NiSO}_{4} \cdot 6 \mathrm{H}_{2} \mathrm{O}(138.65 \mathrm{~g} / \mathrm{l})\end{array}$ & 2.2 & \multirow{3}{*}{1.9} & 0.4 \\
\hline 165 & $\begin{array}{l}\mathrm{H}_{3} \mathrm{BO}_{3}(12.37 \mathrm{~g} / \mathrm{l}) \\
\text { Tiourea }(2 \mathrm{~g} / \mathrm{l}) \\
\text { Vainilla }(2.5 \mathrm{~g} / \mathrm{l})\end{array}$ & 2.1 & & 0.5 \\
\hline 166 & Gluconato de Sodio (4 g/l) & 2.8 & & 1 \\
\hline 167 & $\mathrm{ZnSO}_{4 \cdot 7} \cdot \mathrm{H}_{2} \mathrm{O}(319.97 \mathrm{~g} / \mathrm{l})$ & 3.2 & \multirow{3}{*}{1.25} & 1 \\
\hline 168 & $\begin{array}{l}\mathrm{NiSO}_{4} \cdot 6 \mathrm{H}_{2} \mathrm{O}(233.41 \mathrm{~g} / \mathrm{l}) \\
\mathrm{H}_{3} \mathrm{BO}_{3}(12.37 \mathrm{~g} / \mathrm{l}) \\
\text { Tiourea }(2 \mathrm{~g} / \mathrm{l})\end{array}$ & 3.56 & & 2 \\
\hline 169 & $\begin{array}{l}\text { Vainilla }(2.5 \mathrm{~g} / \mathrm{l}) \\
\text { Alcohol Polivinilico }(5 \mathrm{~g} / \mathrm{l})\end{array}$ & 3.5 & & 3 \\
\hline 170 & & 3.24 & \multirow{5}{*}{1.25} & 0.4 \\
\hline 171 & $\begin{array}{l}\mathrm{ZnSO}_{4} \cdot 7 \mathrm{H}_{2} \mathrm{O}(319.97 \mathrm{~g} / \mathrm{l}) \\
\mathrm{NiSO}_{4} \cdot 6 \mathrm{H}_{2} \mathrm{O}(233.41 \mathrm{~g} / \mathrm{l})\end{array}$ & 3.6 & & 0.5 \\
\hline 172 & $\begin{array}{l}\mathrm{H}_{3} \mathrm{BO}_{3}(12.37 \mathrm{~g} / \mathrm{l}) \\
\text { Tiourea }(2 \mathrm{~g} / \mathrm{l})\end{array}$ & 3.4 & & 1 \\
\hline 173 & $\begin{array}{l}\text { Vainilla }(3.75 \mathrm{~g} / \mathrm{l}) \\
\text { Alcohol Polivinilico }(5 \mathrm{~g} / \mathrm{l})\end{array}$ & 3.1 & & 2 \\
\hline 174 & & 3.58 & & 3 \\
\hline 175 & $\mathrm{ZnSO}_{4} \cdot 7 \mathrm{H}_{2} \mathrm{O}(319.97 \mathrm{~g} / \mathrm{l})$ & 2.9 & \multirow{4}{*}{1.25} & 0.4 \\
\hline 176 & $\begin{array}{l}\mathrm{NiSO}_{4} \cdot 6 \mathrm{H}_{2} \mathrm{O}(233.41 \mathrm{~g} / \mathrm{l}) \\
\mathrm{H}_{3} \mathrm{BO}_{3}(12.37 \mathrm{~g} / \mathrm{ll} \\
\text { Tiourea }(3 \mathrm{~g} / \mathrm{l})\end{array}$ & 2.6 & & 0.5 \\
\hline 177 & Vainilla $(2.5 \mathrm{~g} / \mathrm{L})$ & 2.76 & & 0.75 \\
\hline 178 & Alcohol Polivinilico $(5 \mathrm{~g} / \mathrm{l})$ & 2.6 & & 1 \\
\hline
\end{tabular}




\begin{tabular}{|c|c|c|c|c|}
\hline Prueba & $\begin{array}{c}\text { Relación } \\
\text { molar } \\
\mathrm{Zn} / \mathrm{Ni}\end{array}$ & $\mathrm{J} \mathrm{A} / \mathrm{dm}^{2}$ & $\% \mathrm{Ni}$ & $\mathrm{pH}$ \\
\hline 233 & 0.19 & 6 & 9.8 & 1 \\
\hline 232 & 0.19 & 5 & 4.56 & 1 \\
\hline 235 & 0.19 & 6 & 3.15 & 1 \\
\hline 234 & 0.19 & 5 & 3.32 & 1 \\
\hline 229 & 0.19 & 6 & 6.93 & 1 \\
\hline 228 & 0.19 & 5 & 7.04 & 1 \\
\hline 231 & 0.19 & 6 & 2.96 & 1 \\
\hline 230 & 0.19 & 5 & 4.52 & 1 \\
\hline 225 & 0.19 & 6 & 3.59 & 1 \\
\hline 224 & 0.19 & 3 & 3.22 & 1 \\
\hline 220 & 0.19 & 2 & - & 1 \\
\hline 222 & 0.19 & 6 & 4.5 & 1 \\
\hline 221 & 0.19 & 3 & 3.34 & 1 \\
\hline 220 & 0.19 & 2 & - & 1 \\
\hline 193 & 0.31 & 2 & 2.89 & 3.35 \\
\hline 195 & 0.31 & 3 & 4.15 & 3.69 \\
\hline 192 & 0.31 & 1.5 & - & 3.67 \\
\hline 194 & 0.31 & 2.5 & - & 3.65 \\
\hline 149 & 1.25 & 1.5 & 2.84 & 3.50 \\
\hline 150 & 1.25 & 2 & 2.13 & 3.60 \\
\hline 151 & 1.25 & 3 & 1.87 & 3.36 \\
\hline 152 & 1.25 & 4 & 1.71 & 3.22 \\
\hline 175 & 1.25 & 0.4 & 1.23 & 3.20 \\
\hline 176 & 1.25 & 0.5 & 1.33 & 3.36 \\
\hline 177 & 1.25 & 0.75 & 1.32 & 3.50 \\
\hline 178 & 1.25 & 1 & 1.19 & 3.50 \\
\hline 145 & 1.9 & 1.5 & 2.17 & 3.49 \\
\hline 146 & 1.9 & 2 & 1.60 & 3.57 \\
\hline 147 & 1.9 & 3 & 1.53 & 3.31 \\
\hline 148 & 1.9 & 4 & 1.32 & 3.47 \\
\hline 179 & 1.9 & 0.4 & 1.16 & 3.00 \\
\hline 180 & 1.9 & 0.5 & 1.26 & 3.00 \\
\hline 181 & 1.9 & 0.75 & 1.10 & 2.70 \\
\hline 182 & 1.9 & 1 & 1.28 & 2.80 \\
\hline 188 & 2.5 & 2 & 3.06 & 3.75 \\
\hline 189 & 2.5 & 3 & 2.92 & 3.80 \\
\hline
\end{tabular}

Las pruebas de codeposición iniciales fueron dirigidas para establecer la presencia de Ni en la película y por lo tanto los análisis químicos iniciales fueron de tipo cualitativo, sólo para verificar la presencia del DOI: https://doi.org/10.21754/tecnia. 10.21754/tecnia.v21i2.1033 níquel en el recubrimiento, estas pruebas se realizaron utilizando una marcha analítica [20], que incluía un ataque con ácido nítrico al 10\%, a las limaduras del recubrimiento de la probeta, luego el uso de peróxido de hidrógeno al 3\% para oxidar el $\mathrm{Ni}+1$ a $\mathrm{Ni}+2$, y luego una neutralización hasta $\mathrm{pH} 4$ aproximadamente, para finalmente usar el reactivo dimetilglioxima para la identificación cualitativa del níquel, manifestado por un tono rojo a rosado, estas prueba sirvieron para dirigir los ensayos iniciales.

El análisis cuantitativo de la composición de Zn/Ni en el recubrimiento se usó absorción atómica, el procedimiento iniciaba con el desprendimiento como limaduras de la capa electrodepositada sobre el metal base (acero comercial), luego un ataque con $150 \mathrm{~mL}$ de una solución de $1.2 \mathrm{M}$ de $\mathrm{HNO}_{3}$, a $90{ }^{\circ} \mathrm{C}$ por 30 minutos, para disolver totalmente la muestra.

Las densidades de corriente eléctrica iniciales que se usaron estuvieron entre 0.5 a $3.0 \mathrm{~A} / \mathrm{dm} 2$, para todos los baños a probar, debido a que es lo que se usa en la industria para recubrimientos de zinc [18], no consiguiendo los resultados esperados en apariencia y composición de $\mathrm{Zn} / \mathrm{Ni}$ en el recubrimiento, como se observa en la tabla 2, que además incluye los reportes de absorción atómica de la composición de $\mathrm{Ni}$ en la película.

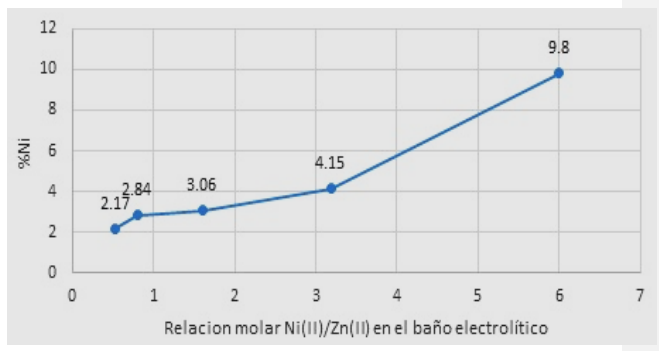

Fig. 4. Efecto de la relación molar de Ni/Zn del baño en \% de Ni en el recubrimiento

Las relaciones molares de $\mathrm{Zn} / \mathrm{Ni}$ de $0.62,1$.

$25,1.9,2.5,3.0$ y 6.0 , que se usaron en los baños de sulfato se consideraron densidades de corriente de 1.5, 2. 3 y $4 \mathrm{~A} / \mathrm{dm}^{2}$, y parte de estos datos se reporta en la tabla II. De este grupo de pruebas se estableció que los baños con una relación molar de 0.19 y $1.5 \mathrm{de} \mathrm{Zn} / \mathrm{Ni}$, se usaran densidades de corriente mayores de $4 \mathrm{~A} / \mathrm{dm}^{2}$ y fueron las que tuvieron mejores resultados, como se observa en tabla II, estos resultados provocó que se usaran densidades de corrientes más altas como de $5 \mathrm{y}$ $6 \mathrm{~A} / \mathrm{dm}^{2}$, para ello además se utilizó diferentes proporciones de corriente variando entre 66/34, 70/30, 80/20 y 90/10 para los ánodos de níquel y zinc respectivamente, se repitieron las pruebas para Revista TECNIA Vol.31 N² Junio-Diciembre 2021 
verificar la consistencia de los resultados y estas probetas fueron evaluadas en su contenido de $\mathrm{Zn}$ y Ni por absorción atómica y esto se evidencia en la tabla III.

Tabla III

\% de Ni y eficiencia catódica.

\begin{tabular}{ccccc}
\hline Prueba & $\mathrm{j}\left(\mathbf{A} / \mathbf{d m}^{2}\right)$ & $\% \mathbf{N i}$ & $\% \mathbf{Z n}$ & $\begin{array}{c}\text { Eficiencia } \\
\text { catódica (\%) }\end{array}$ \\
\hline 233 & 6 & 9.8 & 90.2 & 61.9 \\
232 & 5 & 4.56 & 95.44 & 48.24 \\
235 & 6 & 3.15 & 96.85 & 60.33 \\
234 & 5 & 3.32 & 96.68 & 48.77 \\
229 & 6 & 6.93 & 93.07 & 34.13 \\
228 & 5 & 7.04 & 92.96 & 37 \\
231 & 6 & 2.96 & 97.04 & 60.7 \\
\hline
\end{tabular}

Para medir la velocidad de corrosión del recubrimiento $\mathrm{Zn} / \mathrm{Ni}$ se usó la prueba 233 con la que se obtuvo mayor cantidad de níquel $9.8 \%$ en la película recubierta, y esta a su vez se realizó tres ensayos, para diferentes concentraciones de medios como $3.5 \%$ y $5 \%$ de $\mathrm{NaCl}$, según la tabla IV, para el método de RPL y la tabla $V$ se muestra los resultados para ensayos de corrosión con el mismo tipo de probeta, pero con el método de pendientes (rectas) de Tafel o método de intercepto.

Para el cálculo de la velocidad de corrosión primero se empleó el método de las pendientes de Tafel figura 5 , a partir de las curvas de polarización, para ello se tomó partes de la curva en donde el comportamiento es lineal, para poder emplear el método de extrapolación de las pendientes de Tafel. [3,5,7,14,16,18,19]

Tabla IV

Velocidad de corrosión, método RPL.

\begin{tabular}{|c|c|c|c|c|c|c|}
\hline \multicolumn{7}{|c|}{ Método de Extrapolación de las rectas de Tafel, prueba 233} \\
\hline Ensayo & $\begin{array}{c}\text { ba } \\
\text { (V/decade) }\end{array}$ & $\begin{array}{c}\mathrm{bc} \\
\text { (V/decade) }\end{array}$ & $\begin{array}{l}\text { E corr } \\
(\mathrm{mV})\end{array}$ & $\begin{array}{l}\text { I corr } \\
\left(\mathrm{A} / \mathrm{cm}^{2}\right)\end{array}$ & $\begin{array}{l}\text { V corr } \\
\text { (mpy) }\end{array}$ & $\begin{array}{l}\text { Medio } \\
\% \mathrm{NaCl}\end{array}$ \\
\hline 1 & 0.0309 & 0.07910 & -963 & $8.530 \mathrm{E}-06$ & 4.886 & 3.5 \\
\hline 2 & 0.05220 & 0.06590 & -948 & $8.40 \mathrm{E}-06$ & 4.792 & 3.5 \\
\hline 3 & 0.02230 & 0.2602 & -974 & 12.10E-06 & 6.901 & 5.0 \\
\hline 4 & 0.04370 & 0.05620 & -935 & $10.5 \mathrm{E}-06$ & 6.012 & 5.0 \\
\hline
\end{tabular}

Tabla $\mathrm{V}$

Velocidad de corrosión, método rectas de Tafel.

Método Resistencia a la Polarización, prueba 233

\begin{tabular}{lllll}
\hline Ensayo & $\begin{array}{l}\text { E corr } \\
(\mathrm{mV})\end{array}$ & $\begin{array}{c}\text { I corr } \\
\left(\mathrm{A} / \mathrm{cm}^{2}\right)\end{array}$ & $\begin{array}{l}\text { V corr } \\
(\mathrm{mpy})\end{array}$ & $\begin{array}{l}\text { Medio } \\
\% \mathrm{NaCl}\end{array}$ \\
\hline
\end{tabular}

DOI: https://doi.org/10.21754/tecnia. 10.21754/tecnia.v21i2.1033

\begin{tabular}{lllll}
\hline 1 & -991.6 & $1.576 \mathrm{E}-05$ & 8.987 & $3.5 \%$ \\
2 & -901.7 & $1.30 \mathrm{E}-05$ & 5.872 & $3.5 \%$ \\
3 & -921.4 & $4.07 \mathrm{E}-05$ & 7.095 & $5.0 \%$ \\
\hline
\end{tabular}

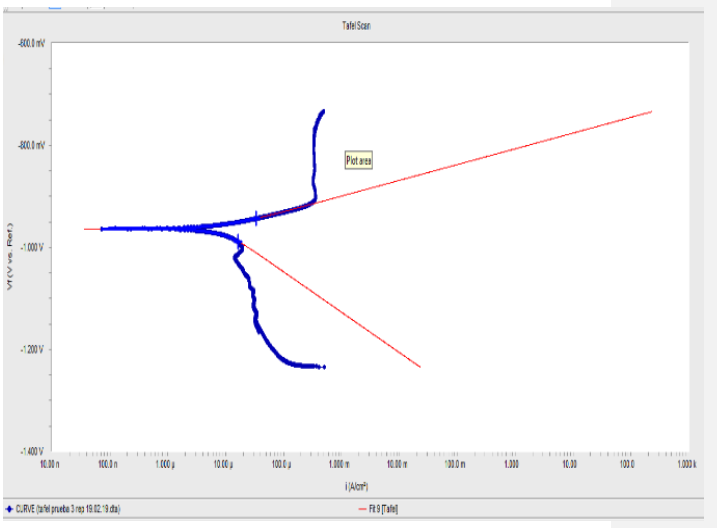

Fig. 5. Pendientes de Tafel, para el cálculo de la velocidad de corrosión E (mV) vs J $\left(\mathrm{A} / \mathrm{dm}^{2}\right)$

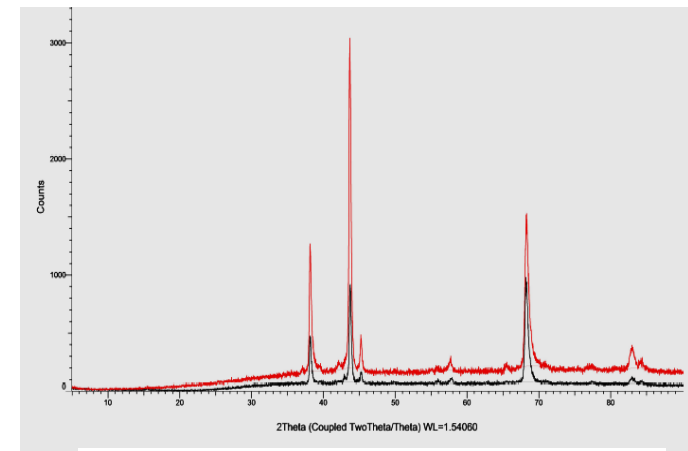

Fig. 6. Difractograma obtenido de dos partes diferentes de una probeta recubierta con la aleación de $\mathrm{Zn} / \mathrm{Ni}$ (9.8\% de Ni en el

Los resultados de las tablas IV y $\mathrm{V}$, se contrastan con una velocidad promedio para un recubrimiento de $\mathrm{Zn}$ puro de $10.55 \mathrm{mpy}$ para $3.5 \% \mathrm{NaCl}$ [21,22], y se observa una mejora evidente.

Las fases presentes en el recubrimiento se analizaron mediante ensayo de difracción de rayos $X$ a dos partes de una probeta (cuyas dimensiones fueron $1 \times 2 \mathrm{~cm}$ ) de la prueba 233. El difractograma de la figura 6 muestran picos de mayor intensidad en $43.7 \mathrm{y}$ 68.2 grados que corresponden a las fases gamma $\gamma$ (Ni5Zn21) y delta $\delta(\mathrm{Ni} 3 \mathrm{Zn} 22)$ respectivamente. Otros picos de menor intensidad en 38.2, 45.3, 57.9 y 83.0 que indican también la presencia de $\mathrm{Zn}$ puro y fase $\eta$ (solución sólida de Ni en Zn). 
Los ensayos de microdureza Vickers y medida del espesor del recubrimiento $\mathrm{Zn} / \mathrm{Ni}$ se hizo sobre la prueba 233 electrodepositada a $6 \mathrm{~A} / \mathrm{dm} 2$, que fue la prueba en la que se obtuvo mayor contenido de níquel en la película, se realizaron a una carga $100 \mathrm{~g}$ por $15 \mathrm{~s}$. en dos partes de la película, mostrado en la tabla VI.

Tabla VI

Ensayo de microdureza HV, en la prueba 233

\begin{tabular}{cccc}
\hline Ensayo & $\begin{array}{c}\text { Microdureza 1 } \\
(\mathrm{HV})\end{array}$ & $\begin{array}{c}\text { Microdureza 2 } \\
(\mathrm{HV})\end{array}$ & $\begin{array}{c}\text { Microdureza } \\
\text { Promedio (HV) }\end{array}$ \\
\hline 1 & 142.7 & 154.8 & 148.75 \\
2 & 140.1 & 152.1 & 146.1 \\
\hline
\end{tabular}

Estos resultados se contrastan con los valores de microdureza para galvanizado en caliente con $70 \mathrm{HV}$ [23] y galvanizado electrolítico de Zn de 45 HV [4], evidenciando una mejora de esta propiedad.

La medida del espesor de la película se hizo dividiendo la probeta (de la prueba 233) de $5 \times 10 \mathrm{~cm}$ en 2 partes de las cuales de obtuvo cupones de $2.5 \times 3 \mathrm{~cm}$, para obtener un espesor promedio. Cada uno de las partes se analizó por separado midiendo el espesor en micras con un microscopio óptico con aumentos de $500 X$ y se obtuvo las micrografías que se observan en las figuras 7 y 8 .

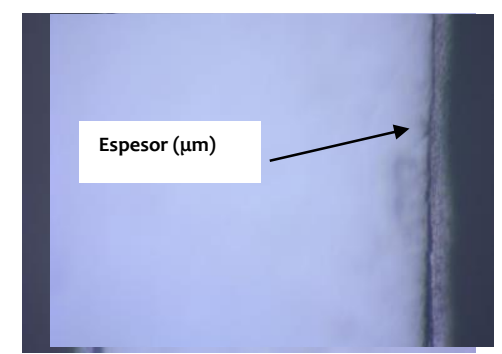

Fig. 8. Fotomicrografía muestra M1 (lado 2), espesor 11 micras, aumentos, $500 \mathrm{X}$

\section{ANÁLISIS DE RESULTADOS}

El mejor baño de sulfato fue $\mathrm{ZnSO} 4.7 \mathrm{H}_{2} \mathrm{O}$ (49 g/l) $\mathrm{NiSO}_{4} .6 \mathrm{H}_{2} \mathrm{O}(268.8 \mathrm{~g} / \mathrm{l}) \mathrm{H}_{3} \mathrm{BO}_{3}(6.185 \mathrm{~g} / \mathrm{l})$ tiourea (0.5 $\mathrm{g} / \mathrm{l})$ gluconato de Sodio $(6 \mathrm{~g} / \mathrm{l})$, corresponde a la prueba 233.

La densidad de corriente que dio mejores resultados fue de $6 \mathrm{~A} / \mathrm{dm} 2$, con una relación de corriente de $90 \%$ para níquel y $10 \%$ para el zinc, dando como resultado un porcentaje de níquel en el recubrimiento de $9.8 \%$.

La mejor relación molar en el baño de $\mathrm{Zn}$ y Ni fue de $19 / 100$, esto es debido al comportamiento anómalo del Ni.

La agitación sobre el baño mejora el recubrimiento incluso a mayor proporción de corriente sobre el ánodo de níquel.

La tiourea en cantidad $0.5 \mathrm{~g} / \mathrm{l}$ funciona muy bien con retardador de zinc, mayores concentraciones disminuye el contenido de níquel.

El gluconato de sodio en cantidad $6 \mathrm{~g} / \mathrm{l}$ funciona muy bien como retardador de zinc.

Existe un efecto sinérgico entre el ácido bórico, tiourea y gluconato de sodio obteniéndose los mejores recubrimientos.

El aumento de la cantidad de tiourea y ácido bórico no mejora el recubrimiento, en apariencia.

La sacarina y el glicerol, ambos como retardadores, no tienen efecto sinérgico con el ácido bórico como abrillantador.

Con vainilla y alcohol polivinílico como retardantes, se obtienen recubrimientos opacos y con manchas “quemadas" con bajo contenido de níquel.

El tipo de morfología del recubrimiento $\mathrm{Zn} / \mathrm{Ni}$ y el difractograma de rayos $X$ confirma la presencia de la fase $\gamma(\mathrm{Ni} 5 \mathrm{Zn} 21)$

La presencia de un $9.8 \%$ de níquel en el recubrimiento mejora en un 300\% la dureza del galvanizado estándar por método electroquímico.

El recubrimiento de zinc y níquel con un contenido de Níquel de $9.8 \%$ en un medio salino $3.5 \% \mathrm{NaCl}$, reduce la velocidad de corrosión en un $47 \%$, y en un medio de $5 \%$ $\mathrm{NaCl}$ reduce la velocidad de corrosión en un $38 \%$.

La presencia de un 9.8\% de níquel aleado con Zinc da un tiempo de vida de hasta el doble con respecto a uno de Zinc puro, aunque el costo se eleva en $37 \%$.

\section{CONCLUSIONES}

Revista TECNIA Vol.31 N² Junio-Diciembre 2021

\section{Comentado [A01]: Equipo utilizafo}

Comentado [aevu2R1]: El equipo está descrito en el ítem 1.1, junto con el resto de equipos utilizados 
- Con el uso de la corriente de $6 \mathrm{~A} / \mathrm{dm}^{2}$, usando $90 \%$ de corriente para el níquel y $10 \%$ para el zinc, se obtuvo un $9.8 \%$ de níquel en la codeposición.

- El baño que estuvo asociado a la mejor codeposición fue $\mathrm{ZnSO}_{4} \cdot 7 \mathrm{H}_{2} \mathrm{O} \quad(49 \mathrm{~g} / \mathrm{l})$ $\mathrm{NiSO}_{4} .6 \mathrm{H}_{2} \mathrm{O}(268.8 \mathrm{~g} / \mathrm{l}) \mathrm{H}_{3} \mathrm{BO}_{3}(6.185 \mathrm{~g} / \mathrm{l})$ tiourea $(0.5 \mathrm{~g} / \mathrm{l})$ gluconato de Sodio $(6 \mathrm{~g} / \mathrm{l})$.

- La agitación permite usar mayor densidad de corriente.

- Se pudo establecer claramente las funciones de retardador y de abrillantador.

- No sólo se obtuvo la composición de 9.8\%, sino además la fase $\gamma$, que asegura un mejor comportamiento contra la corrosión.

- Se obtuvieron mejoras contra los efectos de la corrosión, con $3.5 \%$ de $\mathrm{NaCl}$ se mejoró $47 \%$ y con un medio de $5 \%$ de $\mathrm{NaCl}$ una mejora de $38 \%$.

- La dureza con la codeposición de $\mathrm{Zn} / \mathrm{Ni}$, casi se triplico de $45 \mathrm{HV}$ con un baño de zinc, a $145 \mathrm{HV}$ con la codeposición.

\section{AGRADECIMIENTOS}

Nuestro agradecimiento al Vicerrectorado de Investigación de la Universidad Nacional de Ingeniería, que ha hecho posible el proyecto de investigación, con la subvención del mismo, además a la Facultad de Ingeniería Química y Textil por el uso de sus instalaciones para los ensayos experimentales.

\section{REFERENCIAS}

[1] Z. Mahmud, "Revisión teórica de aleaciones obtenidas por electrodeposición de Zn con elementos del grupo del Fe", Procesos superficiales INTI, vol. 6, pp. 1- 29,Julio, 2016.

[2] M. Gharahcheshmeh y M., Sohi, "Pulse electrodeposition of Zn e Co alloy coatings obtained from an alkaline bath", Mater. Chem. Phys. n 134, pp. 1146-1152, 2012.

[3] M. Tafreshi, S. R. Allahkaram y H. Farhangi “Comparative study on structure, corrosion properties and tribological behavior of pure $\mathrm{Zn}$ and different $\mathrm{Zn-Ni}$ alloy coatings" Mater. Chem. Phys, pp. 263-272, 2016.

[4] M. Abou-Krisha, "Electrochemical studies of zinc-nickel codeposition in sulphate bath", Applied surface science, vol. 252, pp. 1035-1048, 2005.

[5] M. Kwon, D. Jo y S. Cho, "Characterization of the influence of $\mathrm{Ni}$ content on the corrosion resistance of electrodeposited $\mathrm{Zn}-\mathrm{Ni}$ alloy coatings", Surface \& coatings technology, vol. 288, pp. 163-170, 2016 cou s. [6] S. Ghaziof y W. Gao, "Electrodeposition of single gamma phased $\mathrm{Zn}$-Ni alloy coatings from additive-free acidic bath", Applied surface
Science, 2014.

(c) (i)

Los artículos publicados por TECNIA pueden ser compartidos a través de la licencia Creative Commons: CC BY 4.0. Permisos lejos de este alcance pueden ser consultados a través del correo revistas@uni.edu.pe

G. Mohammad y L.i Kordkolaei Naser, of nanocrystalline $\mathrm{Zn} / \mathrm{Ni}$ multilayer coatings from "Electrodeposition of single bath: influences of deposition layers on characteristics of deposits", Applied surface science, vol. 251, pp. 1-34, 2017

[8]S. Ando, "Electrodeposition behavior of $\mathrm{Zn}-\mathrm{Ni}$ alloys produced from sulphate solutions at high current densities", Materials transactions, vol. 57, n. 11, pp. 1908-1914, 2016.
[9] S. Swathirajan, "Electrodeposition of zinc + nickel alloy phases and zinc", J. Electroanal. Chem., vol. 221, pp.211-228, 1987.

[10] S. Fashu, C. Gu, J. Zhang, M. Huang, X. Wang y J. Tu, "Effect of EDTA and $\mathrm{NH}_{4} \mathrm{Cl}$ additives on electrodeposition of $\mathrm{Zn}$ - $\mathrm{Ni}$ films from choline chloride-based ionic liquid", Transactions of nonferrous metals society China, vol. 25, pp. 2054-2064, 2015

[11] G. Sheela,M. Pushpavanam, S. Pushpavanam, "Zinc-nickel alloy electrodeposits for water electrolysis", International journal of hydrogen energy, vol. 27, pp. 627-633, 2002.

[12]Z. Wu, L. Fedrizzi, P. Bonora, "Electrochemical studies of zincnickel codeposition in chloride baths", Surface and coatings technology, vol. 85, pp. 170-174, 1996.

[13]G.A.G. Pedroza, C.A.C De Souza y I. Carlos, "Evaluation of the effect of deposition bath glycerol content on zinc-nickel electrodeposits on carbon steel", Surface \& coatings technology, vol.

[14]T. Byk, T. Gaevskaya y L. Tsybulskaya, "Effect of electrodeposition conditions on the composition, microstructure and corrosion resistance of Zn-Ni alloy coatings", Surface \& coatings technology, vol. 202, pp. 5817-5823, 2008.

[15]F. Elkhatabi, M. Benballa, M. Sarret, "Dependence of coating characteristics on deposition potential for electrodeposited $\mathrm{Zn-Ni}$ alloys" Electrochemical, Acta 44, pp. 1645-1653, 1999.

[16]M. Gavrila, J. P. Millet, H. Mazille y J. Cuntz,"Corrosion behavior of zinc-nickel coatings, electrodeposited on steel” Surface \& coatings technology, vol. 123, pp.164-172, 2000.

[17] L. Felloni, R. Fratesi, E. Quadrini, “Electrodeposition of zinc-nickel alloys from chloride solution" Journal of applied electrochemistry, vol. 17, pp. 574-582, 1987.

[18]R. Fratesi, G. Roventi, “Corrosion resistance of Zn-Ni alloy coatings in industrial production", Surface \& coatings technology, vol. 82, pp. 158-164, 1996.

[19]Mahmud Angela Zulema, Alanis Irene, "Efecto de los asitivos en el desempeño de los recubrimientos de Zinc-Niquel", Procesos superficiales INTI, vol. 6, no. 1, pp. 5-11, jul. 2009

[20] Vogel Arthur I., "Química analítica cuantitativa: Teoría y práctica", vol. 1 y 2, 1era edición, Editorial Kapelusz, Buenos Aires, 1960. [21] K.O. Nayana y T. Venkatesha, "Bright zinc electrodeposition and study of influence of synergistic interaction of additives on coating properties", Journal of Industrial and Engineering Chemestry, vol. 26, pp. 1-9,jun. 2014.

[22] Gharahchesmeh, M. H., "Study of the corrosion behavior of zinc and $\mathrm{Zn}$-Co alloy electrodeposits obtained from alkaline bath using direct current", Materials Chemestry and Physics, vol. 117, no 2-3, pp. 414-421, oct. 2009. [23]Y. Rico y E. Carrasquero, "Efecto de la composición química en el comportamiento mecánico de recubrimientos galvanizados por inmersión en caliente: una revisión", Revista Ciencia y Tecnología, no. 18, pp. $30-392017 . \quad$ Disponible en: https://doi.org/10.17163/ings.n18.2017.04

[24] K. R. Baldwin, M. J. Robinson y C. J. E Smith, "The corrosion resistance of electrodeposited zinc- nickel alloy coatings", Corrosion Science, vol. 35, no. 5-8 , pp. 1267-1272, 1993. [25]R. Fratesi y G. Roventi, “Corrosion resistance of Zn-Ni alloy coat ings in industrial production", Surf.Coat ings Technol, vol. 82,pp. 158$\begin{array}{ll}\text { 164, jul. } & 1996 . \\ \text { [26]Z. A. Mahmud, I. Alanis y C. Moina, “Efeito sinergético de distintos }\end{array}$ aditivos na electrodeposiçao de ligas de Zn- Ni", INTI. Oct. 2000. 206, pp. 2927-2932, 2016. 